\title{
Reporting adverse events in a surgical trial for complex congenital heart disease: The Pediatric Heart Network experience
}

\author{
Lisa Virzi, RN, MS, MBA, ${ }^{a}$ Victoria Pemberton, RNC, MS, CCRC, ${ }^{\mathrm{b}}$ Richard G. Ohye, MD, ${ }^{\mathrm{c}}$ \\ Sarah Tabbutt, MD, PhD, ${ }^{\mathrm{d}}$ Minmin Lu, MS, ${ }^{\mathrm{a}}$ Teresa C. Atz, RN, MSN, ${ }^{\mathrm{e}}$ Teresa Barnard, RN, BSN, ${ }^{\mathrm{f}}$ \\ Carolyn Dunbar-Masterson, BA, BSN, RN, ${ }^{\mathrm{g}}$ Nancy S. Ghanayem, MD, ${ }^{\mathrm{h}}$ Jeffrey P. Jacobs, MD, ${ }^{\mathrm{i}}$ \\ Linda M. Lambert, MSN-cFNP, ${ }^{\mathrm{j}}$ Alan Lewis, MD ${ }^{\mathrm{k}}$ Nancy Pike, RN, PhD, FNP, ${ }^{\mathrm{l}}$ Christian Pizarro, MD, ${ }^{\mathrm{m}}$ \\ Elizabeth Radojewski, RN, CCRP, ${ }^{\mathrm{n}}$ David Teitel, MD, ${ }^{\circ}$ Mingfen Xu, RN, MSN, ${ }^{\mathrm{p}}$ and \\ Gail D. Pearson, MD, $\mathrm{ScD}^{\mathrm{b}}$
}

Objective: The purpose of this analysis was to evaluate a novel strategy for reporting adverse events in the Pediatric Heart Network's randomized surgical trial of systemic-pulmonary artery shunt versus right ventricle-pulmonary artery conduit in infants with hypoplastic left heart syndrome. The strategy was developed to align the reporting process with the needs of a surgical trial while maintaining participant safety.

Methods: Adverse event reporting was analyzed for 2 groups of study subjects: those randomized to a trial arm
during a period in which a standard adverse event reporting system was used (period 1) and those randomized
after institution of a system that focused serious adverse event reporting on 6 sentinel events (period 2). The
analysis encompassed the period from randomization (Norwood surgery) to hospital discharge from stage II sur-
gery. Adverse event rates were compared using a Poisson regression model for the number of events per subject.

Results: From period 1 to period 2, the rate of serious adverse events requiring expedited reporting decreased as expected $(0.42 \mathrm{vs} 0.14 /$ subject/month of follow-up; $P<.001)$. Subjects with a serious (sentinel) adverse event in period 2 had a significantly higher rate of death and cardiac transplantation.

Conclusions: The new adverse event reporting system successfully targeted subjects at highest risk, while decreasing the administrative burden associated with adverse event reports. This methodology may be of benefit in trials evaluating surgical or device-based interventions and in critically ill populations where many common clinical events would qualify as serious adverse events in the context of a drug trial. (J Thorac Cardiovasc Surg 2011;142:531-7)

Little has been published on adverse event (AE) reporting in pediatric research. There is even less experience with $\mathrm{AE}$ reporting in clinical trials in congenital heart disease re-

From the New England Research Institutes a Watertown, Mass; the National Heart,

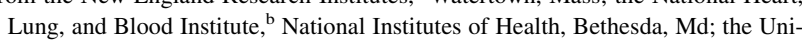
versity of Michigan Medical School, ${ }^{\mathrm{c}}$ Ann Arbor, Mich; the University of Pennsylvania School of Medicine, ${ }^{\mathrm{d}}$ Philadelphia, $\mathrm{Pa}$; the Medical University of South Carolina, ${ }^{\mathrm{e}}$ Charleston, SC; the Cincinnati Children's Hospital Medical Center, ${ }^{\mathrm{f}}$ Cincinnati, Ohio; the Children's Hospital Boston, ${ }^{\mathrm{g}}$ Boston, Mass; the Medical College of Wisconsin and Children's Hospital of Wisconsin, ${ }^{\mathrm{h}}$ Milwaukee, Wis; the University of South Florida College of Medicine, ${ }^{i}$ Tampa, Fla; the Primary Children's Hospital, ${ }^{\mathrm{j}}$ Salt Lake City, Utah; the University of Southern California, ${ }^{\mathrm{k}}$ Los Angeles, Calif; the Children's Hospital Los Angeles, ${ }^{1}$ Los Angeles, Calif; the Jefferson Medical College ${ }^{\mathrm{m}}$ Philadelphia, Pa; The Hospital for Sick Children, ${ }^{\mathrm{n}}$ Toronto, Ontario, Canada; the University of California San Francisco, ${ }^{\circ}$ San Francisco, Calif; and the Duke University Medical Center, ${ }^{\mathrm{p}}$ Durham, NC

For the Pediatric Heart Network Investigators, see Appendix 1.

Supported by U01 grants from the National Heart, Lung, and Blood Institute (HL068269, HL068270, HL068279, HL068281, HL068285, HL068292, HL068290, HL068288, HL085057)

ClinicalTrials.gov number, NCT00115934.

Disclosures: Authors have nothing to disclose with regard to commercial support. Received for publication Sept 16, 2010; revisions received Sept 16, 2010; accepted

for publication Nov 26, 2010; available ahead of print March 14, 2011.

Address for reprints: Victoria Pemberton, RNC, MS, CCRC, 6701 Rockledge Dr,

Room 8102, Bethesda, MD 20892 (E-mail: pembertonv@nhlbi.nih.gov). 0022-5223/\$0.00

Published by Elsevier Inc. on behalf of The American Association for Thoracic Surgery

doi:10.1016/j.jtcvs.2010.11.052 search, and there is no regulatory guidance specific to conducting clinical research in critically ill children. Recent publications have standardized the definitions of a number of complications and AEs for patients undergoing treatment for congenital and pediatric heart diseases,${ }^{1,2}$ but few, if any, publications examine strategies for reporting and grading the severity of these complications and AEs in pediatric cardiac surgical trials.

The Pediatric Heart Network (PHN) was established by the National Heart, Lung, and Blood Institute (NHLBI), National Institutes of Health (NIH), in 2001 to conduct multicenter studies in children with congenital and acquired heart disease. ${ }^{3}$ Early PHN protocols ${ }^{4,5}$ effectively used standard principles for reporting AEs that adhered to the criteria for defining serious AEs (Table 1). These general principles are established by the International Conference on Harmonisation, a body of clinical trial guidance derived from the collaborative efforts of the United States, European Union, and Japan and implemented by the federal Office of Human Research Protections and the Food and Drug Administration. ${ }^{6,7}$ Coding dictionaries or classification systems are used to augment this guidance and provide a standardized approach to reporting AEs in clinical studies. The PHN used the Common Terminology Criteria for Adverse 


\author{
Abbreviations and Acronyms \\ $\mathrm{AE} \quad=$ adverse event \\ CTCAE $=$ Common Terminology Criteria for \\ Adverse Events \\ DCC $=$ Data Coordinating Center \\ DSMB $=$ Data and Safety Monitoring Board \\ IRB = institutional review board \\ NHLBI = National Heart, Lung, and Blood \\ Institute \\ NIH = National Institutes of Health \\ PHN = Pediatric Heart Network \\ SAE $=$ serious adverse event \\ SVR $=$ Single Ventricle Reconstruction trial
}

Events (CTCAE), version 3.0, developed by the National Cancer Institute, NIH. These principles and tools provided standard definitions of AEs and serious adverse events (SAEs), a system of severity scoring, and reporting guidelines, designed particularly for drug trials.

The limitations of the standard AE reporting approaches became apparent during the PHN's Infant Single Ventricle Trial, which compared treatment with enalapril to placebo in critically ill infants with single ventricle physiology., 8,9 There were not enough appropriate pediatric CTCAE codes to cover the complexity of this trial population, and the CTCAE severity scale was not developed for trials in critically ill infants. Discussions, training, and close monitoring for the duration of the trial were implemented to refine our approach to $\mathrm{AE}$ reporting.

Then in May 2005, the PHN launched the Single Ventricle Reconstruction (SVR) Trial, a randomized trial comparing a systemic-pulmonary artery shunt versus a right ventricle-pulmonary artery conduit in infants with hypoplastic left heart syndrome undergoing initial surgical palliation. $^{10,11}$ The SVR trial began with a standard AE reporting framework modified on the basis of lessons learned in the Infant Single Ventricle Trial, but it soon became clear that an AE reporting system based on the standard strategy for drug studies was inappropriate for this surgical trial. The specific challenge was that many clinical events that are common in the postoperative period qualify as serious AEs in the context of a drug trial; thus the standard reporting paradigm resulted in excessive reporting while providing a disproportionately small amount of useful safety information. Moreover, the large number of reports generated considerable administrative workload for individual investigators, study coordinators, and institutional review boards (IRBs)/ research ethics boards as well as the NIH, the data coordinating center, the medical monitor, and the Data and Safety Monitoring Board (DSMB).
To enhance the ability to recognize clinically meaningful events, PHN investigators turned to the sentinel event concept. A sentinel event, as defined by the Joint Commission on Accreditation of Healthcare Organizations, is an unexpected occurrence involving death or serious physical or psychological injury, or the risk thereof, that signals the need for immediate investigation and response. ${ }^{12}$ In a research context, sentinel perinatal events have been identified that predict patterns of brain injury. ${ }^{13}$

The purpose of this analysis was to compare two AE reporting methods used in the SVR trial to assess the accuracy of predicting the most severe adverse outcomes in the study population and the associated changes in reporting burden.

\section{PATIENTS AND METHODS \\ Patient Population}

The design of the SVR trial has been described ${ }^{8}$ and the main results reported. ${ }^{9}$ Neonates with hypoplastic left heart syndrome and other single right ventricle malformations were enrolled at 8 main PHN clinical sites and 7 SVR auxiliary sites. Subjects were randomized to receive a Norwood procedure with either a modified right Blalock-Taussig shunt or right ventricle-pulmonary artery conduit to provide pulmonary blood flow. The primary end point was death or transplant at 12 months. The protocol for the SVR trial was approved by each center's IRB, and written informed consent was obtained from a parent or guardian.

\section{Safety Monitoring and AE Reporting Processes}

The SVR trial protocol underwent review by an independent DSMB appointed by NHLBI to oversee all PHN studies and was subsequently approved by the IRBs at all sites, including the PHN Data Coordinating Center (DCC). AEs were recorded and submitted by the site team via a secure, web-based electronic data capture system to the DCC, with immediate reporting of SAEs to the DCC, NHLBI, and the independent PHN medical monitor, a pediatric cardiologist with expertise in cardiac critical care. Sites reported AEs/SAEs to their IRBs as dictated by local requirements. The DSMB reviewed study data every 6 months, and a summary of this review was sent to the local IRB to ensure that all participating centers were informed of any pertinent safety findings.

SAEs were initially defined in the SVR trial as events that met the criteria in Table 1. In this phase of the SVR trial, May 2005 to January 2007, referred to here as period 1 , each $\mathrm{AE}$ and $\mathrm{SAE}$ was recorded and submitted to the DCC. The events were categorized in standard fashion as to the degree of relatedness to the trial intervention. The site investigator categorized the response to the $\mathrm{AE}$ as surgical or catheter intervention, medical therapy, or none; the clinical outcome was characterized as resolved or stabilized.

A revised approach, developed by the SVR Adverse Events Subcommittee, was implemented from February 2007 to trial end in October 2009 (period 2). The SVR AE subcommittee included congenital heart surgeons, pediatric cardiologists and cardiac intensivists, study coordinators, the AE coordinator from the DCC, and NHLBI staff. The subcommittee reviewed the limited published literature and obtained information from pediatric investigators who had been involved in previous operative and perioperative surgical trials. ${ }^{14-16}$ An AE framework was established consistent with the concept of the sentinel event in patient safety literature.

Six "sentinel" SAEs, considered sufficiently serious for the SVR trial population, were identified (Table 2) and triggered expedited reporting. The frequency of these events was known from published retrospective outcome reports on neonates with hypoplastic left heart syndrome after Norwood palliation, ${ }^{17-19}$ so there was a precedent for determining the number of reported events. Following standard practice, in addition to 
TABLE 1. Criteria for definition of SAEs during period 1, SVR trial Criteria for definition of SAEs, SVR trial

- Fatal

- Life-threatening

- Severely or permanently disabling

- Necessitates significant intervention, such as major surgery, to prevent permanent impairment of a body function or permanent damage to a body structure

- Necessitates or prolongs hospital admission

- PI, medical monitor, or DSMB considers the event to be an SAE

$S A E$, Serious adverse event; $S V R$, Single Ventricle Reconstruction; PI, principal investigator; $D S M B$, Data and Safety Monitoring Board.

these 6 events, any other event that the site principal investigator considered to be an SAE was also reported in expedited fashion. Death, 1 of the 6 sentinel SAEs, was also a primary study end point and required a separate reporting format to the DSMB to preserve the statistical integrity of the final trial data analysis.

During period 2, all events that were not sentinel SAEs were captured in the trial database as complications rather than AEs, using a standardized code list organized by organ system that was developed for this purpose (Table 3). Complications were recorded on an abbreviated case report form, which replaced the detailed AE reporting forms previously used. The SVR trial protocol was amended to reflect these changes, approved by the DSMB and NHLBI, and approved by the IRB at each site. Training sessions were held to familiarize study personnel with the new procedures, and the DCC AE coordinator worked individually with sites to ensure a smooth transition on the effective date of the change.

\section{Data Analysis}

Data analysis was performed to evaluate the effectiveness of the new approach in achieving the goals of facilitating the detection of important safety end points and decreasing the volume of expedited reporting of events. Data were analyzed for 2 groups of study subjects: those randomized during period 1 (May 2005-January 2007) and those randomized during period 2 (February 2007-October 2009). The clinical period of interest for this analysis was from randomization (Norwood surgery) up to hospital discharge from stage II surgery. AEs occurring after the predefined followup window were not included. The data of subjects who had not been discharged from the stage II surgery within the period window were considered incomplete and also excluded from analysis. The number of SAEs, AEs, and complications was determined from the case report forms. $\mathrm{SAE}, \mathrm{AE}$, and complication incidence rates were compared, accounting for varying follow-up time in each $\mathrm{AE}$ reporting period, to normalize the raw numbers of events. A Poisson regression model for the number of events per subject was used to compare the SAE rates of the 2 time periods. In addition to comparing SAE rates, the new reporting system was validated by assessing the rates of death or transplant in the 2 time periods among those for whom an SAE was reported. Event rates in this report are based on the complete trial data set. NHLBI staff participated in the analysis and interpretation of the data for this article.

\section{RESULTS}

During periods 1 and 2, a total of 247 and 224 subjects were randomized and followed up as described earlier. Average follow-up time during period 1 was $3.9 \pm 2.3$ months (median, 4.3 months) compared with $4.7 \pm 2.3$ months (median, 4.8 months) during period 2; the difference results from normalizing the raw numbers of events. In period 1 , $81 \%$ of subjects had an SAE reported, compared with
TABLE 2. SVR sentinel SAEs during period 2 SVR Sentinel SAEs

\section{- Death}

- Acute shunt failure

- Cardiac arrest requiring CPR and medications

- Cardiopulmonary insufficiency requiring ECMO

- Cardiovascular reoperation (unplanned)

- Necrotizing enterocolitis requiring laparotomy

$S V R$, Single Ventricle Reconstruction; $S A E$, serious adverse event; $C P R$, cardiopulmonary resuscitation; $E C M O$, extracorporeal membrane oxygenation.

$48 \%$ in period 2 (Table 4). From period 1 to period 2, the number of reported SAEs decreased by $64 \%$, and the SAE incidence decreased by $67 \%$. This represented a significant drop (by design) in the SAE rate $(0.42$ vs 0.14 events/subject/month of follow-up; $P<.001)$. The incidence of complications in period 2 was 1.0 event/subject/ month of follow-up.

The 1-year death/transplant rate associated with patients who had a sentinel SAE reported under the new system was 1.4 times higher $(66 \%$ vs $46 \% ; P=.001)$ than the rate associated with patients who had an SAE reported under the original system, which included a broader range of clinical AEs. The 1-year death/transplant rates were similar (5\% vs $3 \% ; P=.484)$ for patients who had only a nonserious $\mathrm{AE}$ (period 1 designation) and those who had only a complication (period 2 designation).

Because the number of events reported as SAEs decreased $64 \%$ from 406 in period 1 to 147 in period 2 (Table 4), the number of case report forms submitted to the DCC also decreased by two-thirds. The information requested in the SAE forms did not change from period 1 to period 2.

\section{DISCUSSION}

Standard AE reporting practices for drug trials were inadequate in this randomized cardiovascular surgical trial of fragile neonates. The standard perioperative course for these patients includes many clinical states that would represent SAEs in the CTCAE scoring system. This raised concerns about the ability to detect any single critical safety signal in the large stream of reported data and resulted in a significant reporting burden.

We found that changing to a sentinel SAE reporting strategy allowed us to identify those patients with the highest risk of death or transplant, as exemplified by the fact that rate of death and transplant among patients with a sentinel SAE was significantly higher than in patients with an SAE under the standard reporting system. This finding was an expected outcome of the change in reporting, because under the new system, we were distilling SAE reporting to represent only the most serious, life-threatening events.

When comparing patients who had only nonserious events (called AEs in period 1 and complications in period 
TABLE 3. Code list complications

\section{Cardiac general}

Arrhythmia (recorded if requires medication or treatment)

- Atrial fibrillation

- Atrial flutter

- Supraventricular tachycardia

- Junctional ectopic tachycardia

- Sinus node dysfunction (requiring pacing)

- Atrioventicular block (second or third degree)

- Ventricular tachycardia

- Ventricular fibrillation

Mediastinum

- Hemopericardium (requiring intervention, includes postoperative mediastinal hemorrhage)

- Pericardial effusion (requiring drainage)

- Postpericardiotomy syndrome (requiring treatment)

Cardiac performance

- Hypotension ( $<40 \mathrm{~mm} \mathrm{Hg}$ for neonates; $<50 \mathrm{~mm} \mathrm{Hg}$ after stage II surgery)

- Hypertension (requiring long-term therapy, ie, $>30$ days after discharge, therapy should be at therapeutic doses and specifically prescribed for the treatment of hypertension)

- RV dysfunction (requiring escalation or initiation of therapy, not to include immediate postoperative dysfunction routinely associated with cardiopulmonary bypass)

- Semilunar valve insufficiency or stenosis (requiring treatment initiation or escalation)

- Atrioventricular valve insufficiency or stenosis (requiring treatment initiation or escalation)

- Prosthetic valve dysfunction

Great vessels

- Superior vena cava stenosis (anatomic, symptomatic, "superior vena cava syndrome")

- Superior vena cava occlusion

- Inferior vena cava occlusion

- Other cardiovascular

Respiratory

- Chronic respiratory failure (intubated for $>2$ weeks after surgery)

- Chylothorax (postoperative accumulation of chylous fluid in the pleural space requiring intervention whether by evacuation, dietary change, and/or medical treatment)

- Hemothorax (requiring drainage)

- Phrenic nerve injury/diaphragmatic paralysis (newly elevated diaphragm on chest $\mathrm{X}$-ray film)

- Pleural effusion (requiring drainage $>7$ days after surgery, other)

- Pneumothorax (requiring tube insertion)

- Tracheal injury

- Vocal cord injury (direct visualization)

- Airway obstruction (requiring a significant intervention)

- Hypoxia (requiring readmission or escalation of care)

- Other respiratory

Neurologic

- Choreoathetosis/posturing (moderate involuntary movements interfering with function)

- Coma

- Intracranial bleeding (confirmed by imaging)
TABLE 3. Continued

- Seizure(s) (confirmed by electroencephalogram or obvious motor signs)

- Stroke (confirmed by imaging study)

- Hydrocephalus (report if CTCAE grade > 2)

- Neurologic deficit persisting at discharge not attributed to any of the above diagnoses

- Other neurologic

Gastrointestinal

- Direct bilirubin $>4 \mu \mathrm{mol} / \mathrm{L}$

- Liver failure (AST, ALT, or GGT > 500 U/L)

- Necrotizing enterocolitis, confirmed (pneumatosis or free air)

- Necrotizing enterocolitis, suspected (NPO, antibiotics started)

- Other esophageal or bowel perforations not associated with necrotizing enterocolitis

- Upper gastrointestinal bleed, requiring treatment

- Stricture/stenosis (CTCAE > grade 2)

- Other gastrointestinal

Infectious

- Empyema

- Endocarditis

- Gastroenteritis or enteritis

- Line infection, bacterial (positive blood cultures)

- Line infection, fungal (positive blood cultures with initiation of therapy)

- Pneumonia, respiratory infection, viral (requiring the initiation of therapy)

- Mediastinitis/wound infection, deep (requiring incision and drainage; sternal instability)

- Wound infection, superficial (erythema, possible tissue separation and drainage)

- Sepsis, confirmed (positive blood cultures, not line infection)

- Sepsis, clinical with negative cultures

- Urinary tract infection

- Other infection

\section{Renal}

- Acute renal failure (creatinine $>1.5 \mathrm{mg} / \mathrm{dL}(133 \mu \mathrm{mol} / \mathrm{L})$ or tripling of baseline value for $<7$ days; temporary dialysis)

- Chronic renal failure (creatinine $>1.5 \mathrm{mg} / \mathrm{dL}(133 \mu \mathrm{mol} / \mathrm{L})$ or tripling of baseline value for $>7$ days; long-term dialysis)

- Other renal

\section{Hematologic}

- Anemia (hemoglobin $<10 \mathrm{gm} / \mathrm{L}$ )

- Thrombocytopenia (platelets $<50 \times 10^{9} / \mathrm{L}$ )

- Hematoma (CTCAE grade $>2$ )

- Hemorrhage, gastrointestinal (CTCAE grade $>2$; hemepositive stools)

- Hemorrhage, genitourinary (CTCAE grade $>2$ )

- Hemorrhage, pulmonary/upper respiratory (CTCAE grade $>2$ )

- Other hematologic

Vascular

- Thrombus/thromboembolism

- Vascular, other

Other complication

- Other

$R V$, Right ventricular; $C T C A E$, Common Terminology Criteria for Adverse Events; $A S T$, aspartate aminotransferase; $A L T$, alanine aminotransferase; $G G T$, gammaglutamyl transferase; $N P O$, nothing by mouth. 
TABLE 4. Results of changes in $\mathrm{AE}$ reporting from randomization to discharge from stage II hospitalization

\begin{tabular}{lcc}
\hline & Period 1 & Period 2 \\
\hline - Subjects & 247 & 224 \\
- Total follow-up time, mo & 956 & 1060 \\
- Follow-up time, mo mean (SD) & $3.9(2.3)$ & $4.7(2.3)$ \\
- SAEs & 406 & 147 \\
- SAEs incidence, events/subject/month & 0.42 & 0.14 \\
$\quad$ of follow-up* & & \\
- Subjects with SAE, n (\%) & $199(81)$ & $107(48)$ \\
- AEs & 344 & N/A \\
- AE incidence, events/subject/month & 0.36 & N/A \\
of follow-up & & \\
- Complications & N/A & 1,059 \\
- Complication incidence, events/subject/month & N/A & 1.00 \\
of follow-up & & \\
\hline $\begin{array}{l}A E, \text { Adverse event; } S D \text {, standard deviation; } S A E \text {, serious adverse event; } N / A, \text { not ap- } \\
\text { plicable. } * P<.001 \text { for period 1 versus period 2. }\end{array}$ &
\end{tabular}

2 ), we found that these 2 groups were homogeneous with regard to risk, based on their similar rates of death and transplant. Additionally, the events classified as SAEs in period 1 , for which the classification changed to "complication" in period 2, were more similar to nonserious AEs than to SAEs, providing further verification that we had successfully focused the SAE reporting process on the highestrisk events.

The new system also allowed study coordinators and investigators to focus their time and efforts on reporting and responding to the most severe events. The $64 \%$ reduction in the volume of SAEs reported, with no change in the information requested on SAE reports, suggests that study coordinator time for reporting SAEs could have been reduced by as much as two-thirds, although this was not measured directly. Other perceived efficiencies were gained, including a decrease in reporting burden to local IRBs and decreased review time by IRBs, the medical monitor, and the DSMB.

Changing to a system of specified sentinel events also, by design, decreased the variability in what was reported as an SAE across sites. Site investigators, typically congenital heart surgeons or pediatric cardiac intensivists, were understandably accustomed to thinking in terms of a normal postoperative course, as opposed to what would be normal for a healthy nonhospitalized neonate. This led to some sites reporting events or procedures such as gastrostomy tube placement or diaphragm plication as SAEs, while other sites considered the same event or procedure to be part of typical postoperative care and thus did not report it. During period 1, a considerable amount of time was spent attempting to harmonize the types of events reported. This problem was solved with the sentinel SAE strategy used in period 2, because sites were reporting on a small number of predefined events.

There is a large body of literature devoted to patient safety and clinical error prevention designed to minimize morbidity and mortality and to evaluate quality of care.
Ensuring the safety of subjects in clinical trials has received less attention. AE reporting is one of the main tools to assess safety, but the good clinical practice principles that govern the conduct of clinical research were developed primarily for drug trials. Furthermore, most clinical trials have been conducted in adults. It was not until approximately 1 decade ago that pediatric research began to be emphasized as a matter of policy by the NIH and by Congress with the passage of the Food and Drug Administration Modernization Act of 1997, the Best Pharmaceuticals for Children Act (2002), ${ }^{20}$ and the Pediatric Research Equity Act (2003). ${ }^{21}$ We believe that our approach may represent a useful paradigm for future surgical trials, as well as for trials of critically ill participants in general. This approach has already been adopted by another NHLBI-funded trial of therapeutic hypothermia in children after cardiac arrest ${ }^{22}$ (personal communication from Victoria Pemberton, December 28, 2008).

\section{LIMITATIONS}

This was not a prospectively designed comparison, but rather reflects a retrospective evaluation of a practical change made in $\mathrm{AE}$ reporting procedures during the conduct of a clinical trial. Therefore, we do not know whether unmeasured factors could have influenced our results and thus the generalizability of the findings. Nonetheless, our results consistently support the benefits of the change. Furthermore, there is no evidence from these data or other trial monitoring data that there were any untoward effects of the reporting change.

\section{CONCLUSIONS}

In a randomized surgical trial in neonates with complex congenital heart disease, expedited reporting of AEs based on existing methods was found to be impractical. An overwhelming volume of data was generated, which not only increased the chance of error, but also risked obscuring true indicators of concern. Thus, a novel methodology was developed to effectively and efficiently capture appropriate events. This new technique relies on monitoring a small number of clearly defined sentinel SAEs and resulted in comparable identification of clinically relevant events while decreasing the administrative reporting burden. This methodology may be of benefit in trials evaluating surgical or device-based interventions and in critically ill populations where many common clinical events would qualify as SAEs in the context of a drug trial. Additional research on this methodology as well as on pediatric AE reporting in general is needed.

We thank Christine Kang, MDiv, for assisting in the preparation and submission of this manuscript.

\section{References}

1. Jacobs JP, Mavroudis C, Jacobs ML, Maruszewski B, Tchervenkov CI, LacourGa.yet FG, et al. What is operative mortality? Defining death in a surgical 
registry database: a report of the STS Congenital Database Taskforce and the Joint EACTS-STS Congenital Database Committee. Ann Thorac Surg. 2006; 81:1937-41.

2. Jacobs JP, Benavidez OJ, Bacha EA, Walters HL, Jacobs ML. The nomenclature of safety and quality of care for patients with congenital cardiac disease: a report of the Society of Thoracic Surgeons Congenital Database Taskforce Subcommittee on Patient Safety. Cardiol Young. 2008;18(Suppl. 2):81-91.

3. Mahony L, Sleeper LA, Anderson PA, Gersony WM, McCrindle BW, Minich LL, et al. The Pediatric Heart Network: a primer for the conduct of multicenter studies in children with congenital and acquired heart disease. Pediatr Cardiol. 2006;27:191-8.

4. Anderson PA, Sleeper LA, Mahony L, Colan SD, Atz AM, Breitbart RE, et al. Contemporary outcomes after the Fontan procedure: a Pediatric Heart Network multicenter study. J Am Coll Cardiol. 2008;52:85-98.

5. Newburger JW, Sleeper LA, McCrindle BW, Minich LL, Gersony W, Vetter VL, et al. Randomized trial of pulsed corticosteroid therapy for primary treatment of Kawasaki disease. $N$ Engl J Med. 2007;356:663-75.

6. ICH Steering Committee. ICH harmonised tripartite guideline: Clinical safety data management: definitions and standards for expedited reporting E2A. International Conference on Harmonisation of Technical Requirements for Registration of Pharmaceuticals for Human Use. 1994

7. ICH Steering Committee. ICH harmonised tripartite guideline: Guideline for good clinical practice E6 (R1). International Conference on Harmonisation of Technical Requirements for Registration of Pharmaceuticals for Human Use 1996; Geneva.

8. Hsu DT, Mital S, Ravishankar C, Margossian R, Li JS, Sleeper LA, et al. Rationale and design of a trial of angiotensin-converting enzyme inhibition in infants with single ventricle. Am Heart J. 2009;157:37-45.

9. Hsu DT, Zak V, Mahony L, Sleeper LA, Atz AM, Levine JC, et al. Enalapril in infants with single ventricle: results of a multicenter randomized trial. Circulation. 2010;122:333-40.

10. Ohye RG, Gaynor JW, Ghanayem NS, Goldberg CS, Laussen PC, Frommelt PC, et al. Design and rationale of a randomized trial comparing the Blalock-Taussig and right ventricle-pulmonary artery shunts in the Norwood procedure. J Thorac Cardiovasc Surg. 2008;136:968-75.

11. Ohye RG, Sleeper LA, Mahony L, Newburger JW, Pearson GD, Lu M, et al. Comparison of shunt types in the Norwood procedure for single-ventricle lesions. N Engl J Med. 2010;36:1980-92.

12. The Joint Commission.org [Internet]. Sentinel Event Alert [cited 2010 Apr 7]. Available from: http://www.jointcommission.org/SentinelEvents/

13. Okereafor A, Allsop J, Counsell SJ, Fitzpatrick J, Azzopardi D, Rutherford MA et al. Patterns of brain injury in neonates exposed to perinatal sentinel events. Pediatrics. 2008;121:906-14.

14. Jonas RA, Wypij D, Roth SJ, Bellinger DC, Visconti KJ, du Plessis AJ, et al. The influence of hemodilution on outcome after hypothermic cardiopulmonary bypass: results of a randomized trial in infants. J Thorac Cardiovasc Surg. 2003; 126:1765-74.

15. Tabbutt S, Nicolson SC, Adamson PC, Zhang X, Hoffman ML, Wells W, et al. The safety, efficacy, and pharmacokinetics of esmolol for blood pressure control immediately after repair of coarctation of the aorta in infants and children: a multicenter, double-blind, randomized trial. J Thorac Cardiovasc Surg. 2008;136:321-8.

16. Hoffman TM, Wernovsky G, Atz AM, Kulik TJ, Nelson DP, Chang AC, et al. Efficacy and safety of milrinone in preventing low cardiac output syndrome in infants and children after corrective surgery for congenital heart disease. Circulation. 2003;107:996-1002.

17. Cua CL, Thiagarajan RR, Gauvreau K, Lai L, Costello JM, Wessel DL, et al. Early postoperative outcomes in a series of infants with hypoplastic left heart syndrome undergoing stage I palliation operation with either modified Blalock-Taussig shunt or right ventricle to pulmonary artery conduit. Pediatr Crit Care Med. 2006;7:238-44.

18. Tabbutt S, Dominguez TE, Ravishankar C, Marino BS, Gruber PJ, Wernovsky G, et al. Outcomes after the stage I reconstruction comparing the right ventricular to pulmonary artery conduit with the modified Blalock Taussig shunt. Ann Thorac Surg. 2005;80:1582-90; discussion 90-1.

19. Ashburn DA, McCrindle BW, Tchervenkov CI, Jacobs ML, Lofland GK, Bove EL, et al. Outcomes after the Norwood operation in neonates with critical aortic stenosis or aortic valve atresia. J Thorac Cardiovasc Surg. 2003;125: 1070-82.

20. Eunice Kennedy Shriver National Institute of Child Health \& Human Development [Internet]. Best Pharmaceuticals for Children Act [cited 2010 Apr 7]. Available from: http://bpca.nichd.nih.gov.
21. US Food and Drug Administration [Internet]. Pediatric Drug Development [cited 2010 Apr 7]. Available from: http://www.fda.gov/Drugs/DevelopmentApproval Process/DevelopmentResources/ucm049867.htm.

22. ClinicalTrials.org [Internet]. The THAPCA-OH Study [cited 2010 Apr 7]. Available from: http://clinicaltrials.gov/ct2/show/NCT00878644?term=THAPCA\& rank $=2$.

\section{APPENDIX}

National Heart, Lung, and Blood Institute. Gail Pearson, Victoria Pemberton, Rae-Ellen Kavey,* Mario Stylianou, Marsha Mathis*

Network Chair. University of Texas Southwestern Medical Center, Lynn Mahony

Data Coordinating Center. New England Research Institutes, Lynn Sleeper (PI), Sharon Tennstedt (PI), Steven Colan, Lisa Virzi, Patty Connell,* Victoria Muratov, Lisa Wruck,* Minmin Lu, Dianne Gallagher, Anne Devine,* Julie Schonbeck, Thomas Travison,* David F. Teitel

Core clinical site investigators. Children's Hospital Boston, Jane W. Newburger (PI), Peter Laussen, Pedro del Nido, Roger Breitbart, Jami Levine, Ellen McGrath, Carolyn Dunbar-Masterson, John E. Mayer, Jr, Frank Pigula, Emile A. Bacha, Francis Fynn-Thompson; Children's Hospital of New York, Wyman Lai (PI), Beth Printz,* Daphne Hsu,* William Hellenbrand, Ismee Williams, Ashwin Prakash,* Seema Mital,* Ralph Mosca,* Darlene Servedio,* Rozelle Corda, Rosalind Korsin, Mary Nash; Children's Hospital of Philadelphia, Victoria L. Vetter (PI), Sarah Tabbutt,* J. William Gaynor (Study Co-Chair), Chitra Ravishankar, Thomas Spray, Meryl Cohen, Marisa Nolan, Stephanie Piacentino, Sandra DiLullo, Nicole Mirarchi; Cincinnati Children's Medical Center, D. Woodrow Benson (PI), Catherine Dent Krawczeski, Lois Bogenschutz, Teresa Barnard, Michelle Hamstra, Rachel Griffiths, Kathryn Hogan, Steven Schwartz,* David Nelson, Pirooz Eghtesady; North Carolina Consortium: Duke University, East Carolina University, Wake Forest University, Page A. W. Anderson (PI)—deceased, Jennifer Li (PI), Wesley Covitz, Kari Crawford,* Michael Hines, James Jaggers,* Theodore Koutlas, Charlie Sang, Jr, Lori Jo Sutton, Mingfen Xu; Medical University of South Carolina, J. Philip Saul (PI), Andrew Atz, Girish Shirali, Scott Bradley, Eric Graham, Teresa Atz, Patricia Infinger; Primary Children's Medical Center and the University of Utah, Salt Lake City, Utah, L. LuAnn Minich (PI), John A. Hawkins, Michael Puchalski, Richard V. Williams, Peter C. Kouretas, Linda M. Lambert, Marian E. Shearrow, Jun A. Porter; Hospital for Sick Children, Toronto, Brian McCrindle (PI), Joel Kirsh, Chris Caldarone, Elizabeth Radojewski, Svetlana Khaikin, Susan McIntyre, Nancy Slater; University of Michigan, Caren S. Goldberg (PI), Richard G. Ohye (Study Chair), Cheryl Nowak; Children's Hospital of Wisconsin and Medical

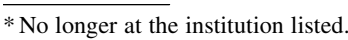


College of Wisconsin, Nancy S. Ghanayem (PI), James S. Tweddell, Kathleen A. Mussatto, Michele A. Frommelt, Peter C. Frommelt, Lisa Young-Borkowski

Auxiliary sites. Children's Hospital Los Angeles, Alan Lewis (PI), Vaughn Starnes, Nancy Pike; The Congenital Heart Institute of Florida (CHIF), Jeffrey P. Jacobs, MD (PI), James A. Quintessenza, Paul J. Chai, David S. Cooper, J. Blaine John, James C. Huhta, Tina Merola, Tracey Grifith; Emory University, Kirk Kanter (PI), William Mahle, Joel Bond,* Jeryl Huckaby; Nemours Cardiac Center, Christian Pizarro, Carol Prospero, Julie Simons, Gina Baffa, Wolfgang A. Radtke; University of Texas Southwestern Medical Center, Ilana Zeltzer (PI), Tia Tortoriello,* Deborah McElroy, Deborah Town
Angiography core laboratory. Duke University, John Rhodes, J. Curt Fudge

Echocardiography core laboratories. Children's Hospital of Wisconsin, Peter Frommelt; Children's Hospital Boston, Gerald Marx.

Genetics core laboratory. Children's Hospital of Philadelphia, Catherine Stolle

Protocol Review Committee. Michael Artman (Chair), Erle Austin, Timothy Feltes, Julie Johnson, Thomas Klitzner, Jeffrey Krischer, G. Paul Matherne

Data and Safety Monitoring Board, John Kugler (Chair), Rae-Ellen Kavey,* Executive Secretary; David J. Driscoll, Mark Galantowicz, Sally A. Hunsberger, Thomas J. Knight, Holly Taylor, Catherine L. Webb 